Received: 2 June 2018

Accepted: 23 October 2018

Published online: 13 November 2018

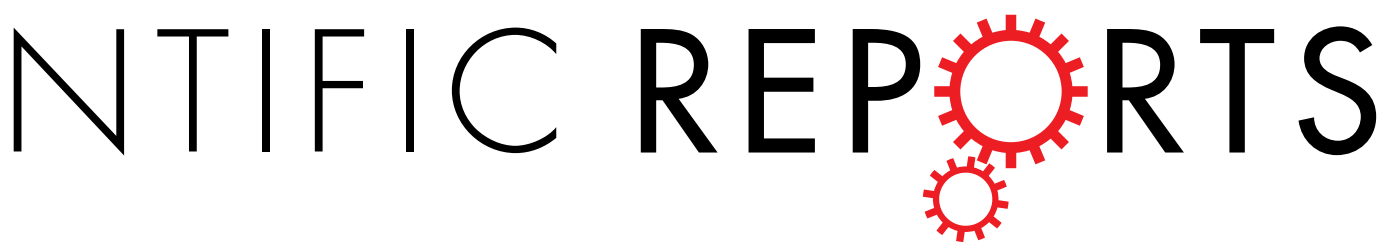

\title{
OPEN Modulating Fundamental Resonance in Capacitive Coupled Asymmetric Terahertz Metamaterials
}

\author{
S. Jagan Mohan Rao $\mathbb{1}^{1}$, Yogesh Kumar Srivastava², Gagan Kumar ${ }^{1} \&$ Dibakar Roy Chowdhury $^{3}$
}

In this work, we experimentally investigate near-field capacitive coupling between a pair of single-gap split ring resonators (SRRs) in a terahertz metamaterial. The unit cell of our design comprises of two coupled SRRs with the split gaps facing each other. The coupling between two SRRs is examined by changing the gap of one resonator with respect to the other for several inter resonator separations. When split gap size of one resonator is increased for a fixed inter-resonator distance, we observe a split in the fundamental resonance mode. This split ultimately results in the excitation of narrow band low frequency resonance mode along with a higher frequency mode which gets blue shifted when the split gap increases. We attribute resonance split to the excitation of symmetric and asymmetric modes due to strong capacitive or electric interaction between the near-field coupled resonators, however blue shift of the higher frequency mode occurs mainly due to the reduced capacitance. The ability of nearfield capacitive coupled terahertz metamaterials to excite split resonances could be significant in the construction of modulator and sensing devices beside other potential applications for terahertz domain.

In the electromagnetic spectrum, terahertz gap exists between microwave and infrared regions and is potentially significant to a variety of applications ranging from medical sciences to engineering ${ }^{1}$. Many natural materials inherently do not respond to terahertz radiation. However in this scenario, metamaterials are quite promising to make devices such as antennas, modulators, next generation high speed communication devices, ultra-sensitive detectors, sensors etc. for terahertz frequencies ${ }^{1-11}$. Metamaterials exhibit many interesting properties such as negative refractive index ${ }^{12}$, perfect focusing ${ }^{13}$, cloaking ${ }^{14,15}$, and resonance modulation in the active and passive modes $^{16-26}$ etc. Fundamentally Metamaterial (MM) is an arrangement of artificial structured elements designed to achieve unusual but desired electromagnetic (EM) properties ${ }^{27-33}$. Typically, split ring resonators (SRRs) are the basic building blocks of metamaterials with lattice constant much smaller than the excitation wavelength ${ }^{34-39}$. In recent times, a lot of emphasis has been given to the fabrication of terahertz metamaterials and realization of terahertz photonic devices. Because of the longer wavelengths of terahertz radiation, it is relatively convenient to fabricate metamaterials at terahertz frequencies. In order to get suitable spectral response from the metamaterial structures, numerous approaches based on metamaterials have been employed which include modification of substrate parameters ${ }^{40,41}$, lumped capacitors or varactors based $\mathrm{MM}^{36}$, usage of liquid crystals ${ }^{42,43}$, ferromagnetic and ferroelectric techniques ${ }^{42}$, microelectromechanical systems (MEMS) based switches ${ }^{44}$, resonance modulation based on near-field interactions between nearest neighbor SRRs ${ }^{45-52}$ etc. In our present study, we have focused on near-field manipulations between nearest neighbor SRRs. Metamaterials in near-field coupled configuration can consist of more than one SRR (meta-atoms) in a unit cell and intelligently manipulating the near-field coupling between these resonators, can have significant impact on the metamaterials responses. Therefore, controlling the near-field coupling in metamaterials is extremely crucial in order to determine its role in designing and fabrication of terahertz photonic components viz. modulators, filters, polarization rotation etc. By nature, near-field coupling in metamaterials could be resistive, capacitive or inductive. Several near-field coupling mechanisms have been studied in recent past using different schemes, however these coupling mechanisms have been extensively

${ }^{1}$ Department of Physics, Indian Institute of Technology Guwahati, Guwahati, 781039, Assam, India. ${ }^{2}$ Division of Physics and Applied Physics, School of Physical and Mathematical Sciences, Nanyang Technological University, Singapore, 637371, Singapore. ${ }^{3}$ Mahindra Ecole Centrale, Jeedimetla, Hyderabad, 500043, Telengana, India. Correspondence and requests for materials should be addressed to D.R.C. (email: dibakarrc@gmail.com) 

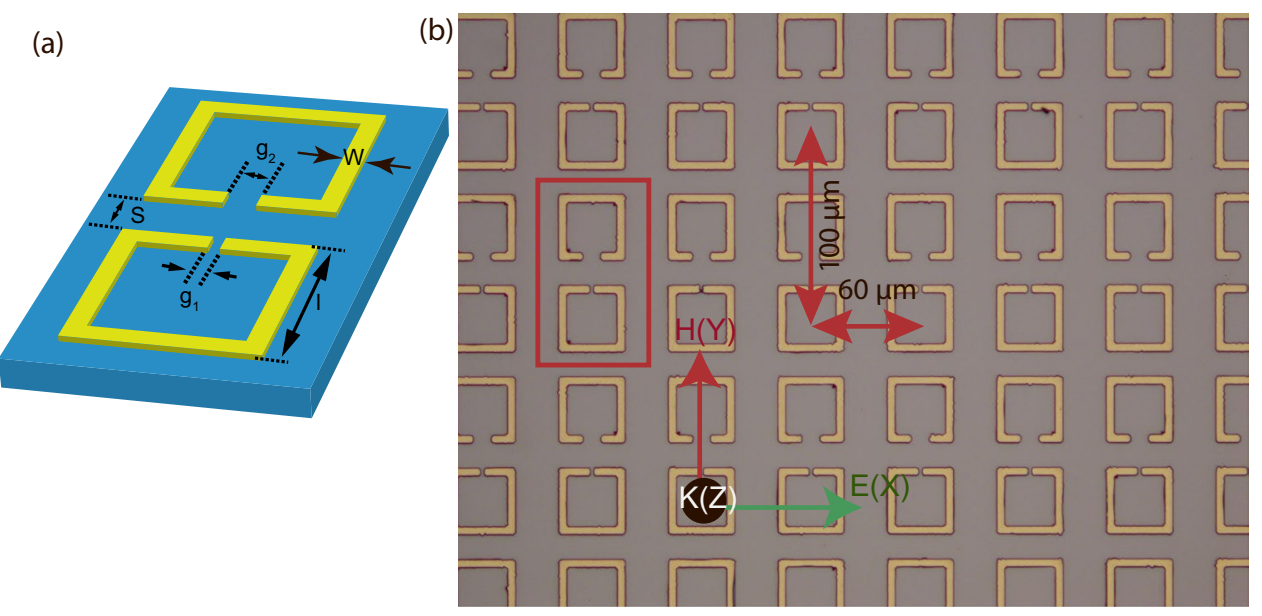

Figure 1. (a) Schematic of the unit cell comprising of two SRRs in a coupled THz metamaterials. The blue regions indicate substrate while the yellow regions represent metallic areas. Each of the SRRs has an outer dimension of $l \times l=36 \times 36 \mu \mathrm{m}$ and gap $\left(g_{1}\right)$ of bottom SRR is $2 \mu \mathrm{m}$, gap of top SRR is $g_{2}\left(g_{2}=2,6,10 \ldots 28 \mu \mathrm{m}\right)$, width (w) of SRR is $4 \mu \mathrm{m}$. ' $\mathrm{S}$ ' represents the separation between two SRRs in Y-direction $(\mathrm{S}=2,6,10$ and $14 \mu \mathrm{m})$. (b) Optical microscope image of fabricated sample.
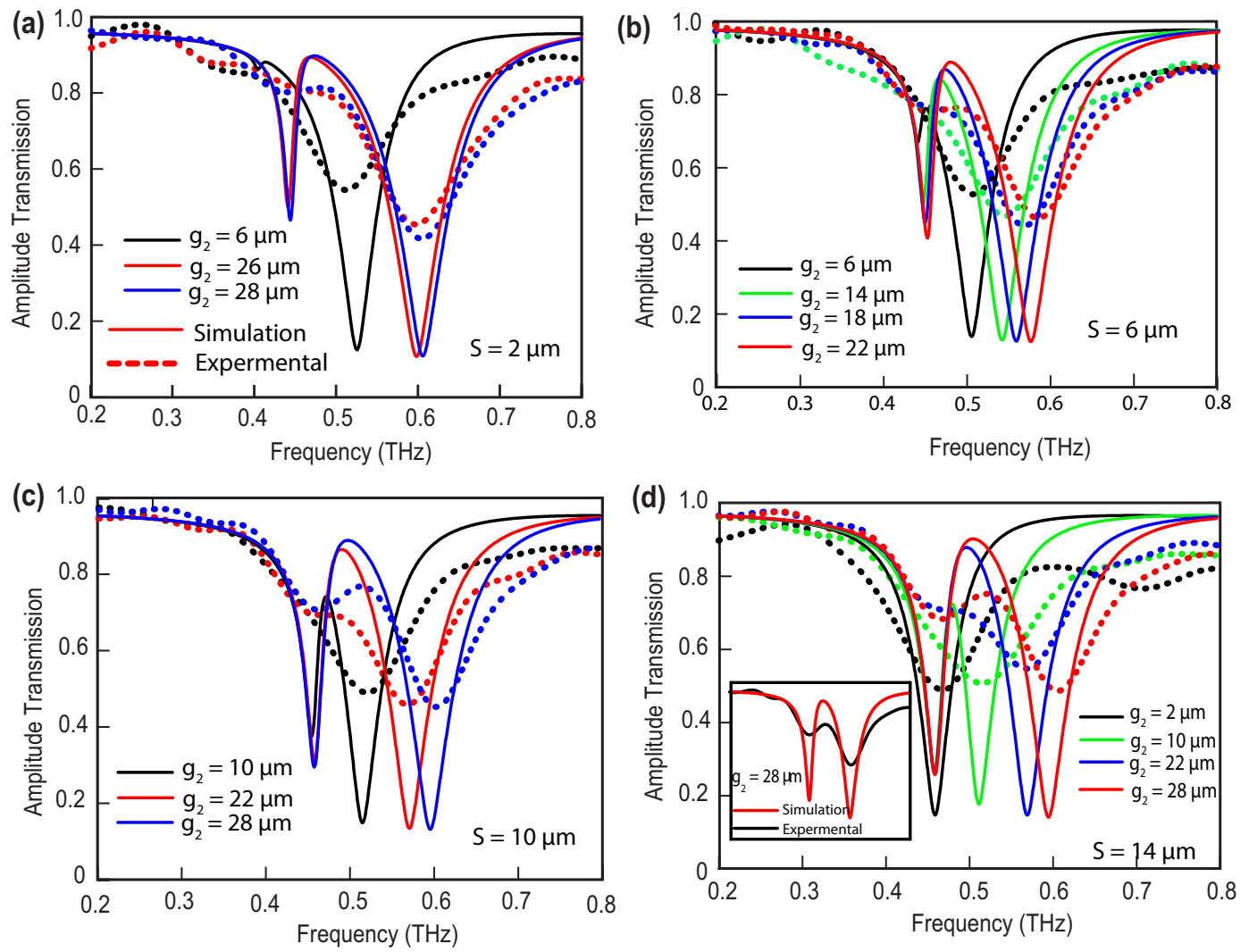

Figure 2. Experimental and numerically calculated amplitude spectra for four different separations. Thick lines represent the simulation results and the dotted lines represents transmission data. $g_{2}=28 \mu \mathrm{m}$ case is enlarged in inset of (d) for elaboration.

electric and magnetic in nature ${ }^{19,20,53,54}$. In our earlier works, we have investigated broadside coupling along with near-filed inductive coupling in planar terahertz metamaterial ${ }^{55}$.

In this paper, we have focused mainly on the capacitive near-field coupling in a terahertz metamaterial. Although, the investigations on near-field coupling in terahertz metamaterials have led to many interesting physics and engineering, but actualization of terahertz devices using such coupling mechanisms require other challenges to be addressed. This includes engineering the near-field capacitive coupling to excite sharp low bandwidth 

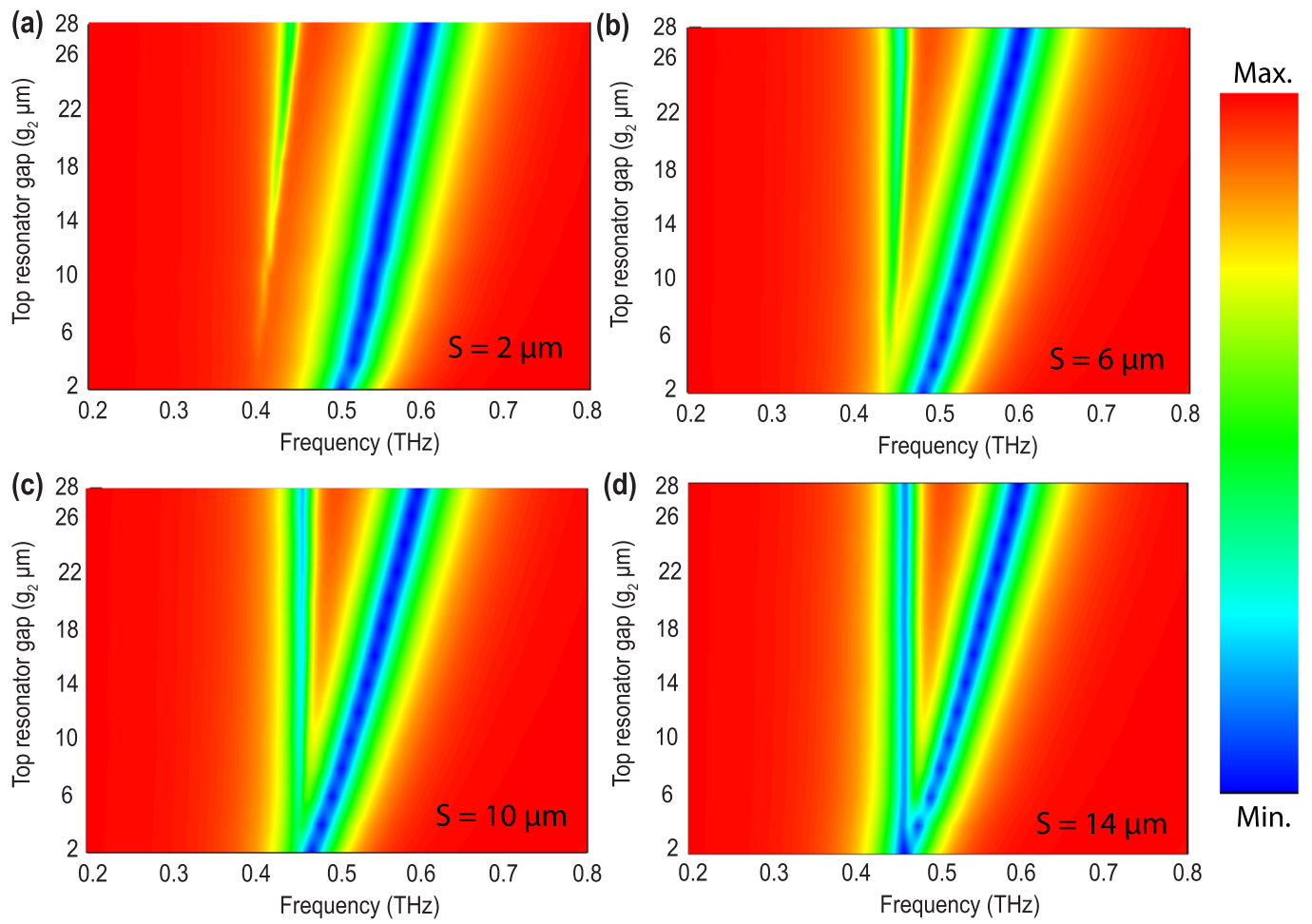

Figure 3. Contour plot of numerically simulated $\mathrm{THz}$ transmittance for four different separation between two SRRs. Color bar shows the magnitude of transmission intensity. (a) Represents contour plots for separations between two SRRs i.e. $S=2 \mu \mathrm{m}$ case. (b-d) represents contour plots for $S=6,10,14 \mu \mathrm{m}$, respectively.

resonance which is not demonstrated earlier to the best of our knowledge. In this context, gap to gap near-field capacitive coupling between adjacent SRRs can result in either blue shift or red shift of the fundamental resonance, based upon the relative positions of the split gap of SRRs ${ }^{56}$. This provides an extra degree of freedom to manipulate and control the phase and amplitude of the terahertz wave propagation. Further, near-field interaction between the adjacent SRRs results in a strong redistribution of energy in comparison to the single SRR giving an additional degree of freedom in designing metamaterials. In this paper, we specifically explore the asymmetric gap to gap near-field capacitive coupling between a pair of SRRs in a coupled unit cell. This is achieved by changing the gap size of one resonator with respect to the other. We have extended our study from strong near-field regime to weak near-field regime. Because of the interactions between the asymmetric split gaps, the asymmetric and symmetric coupled resonance modes are excited which results in low bandwidth and frequency tunable resonance modes.

\section{Results}

Metamaterial design and experimental details. The design of terahertz (THz) metamaterials is crucial in accomplishing the desired electromagnetic response. In our proposed metamaterial configuration, metamolecule (unit cell) is comprised of two split ring resonators (SRRs) with gaps facing each other. A schematic of the proposed configuration is shown in Fig. 1(a). We have assumed silicon as the substrate. The unit cell is chosen to be sufficiently bigger in size $\left(P_{x}=60 \mu \mathrm{m}\right.$ and $\left.P_{y}=100 \mu \mathrm{m}\right)$ compared to the metamolecule or SRR pair in order to avoid any undesired near-field couplings between the neighboring unit cells. In such a metamolecule design, both the SRRs i.e. top and bottom as depicted in the figure are $36 \times 36 \mu \mathrm{m}$ in length and breadth with an aluminum layer of thickness of $200 \mathrm{~nm}$ deposited at the top side. The capacitive gap and line width of the bottom resonators are assumed to be $g_{1}=2 \mu \mathrm{m}$ and $w=4 \mu \mathrm{m}$, respectively. The above mentioned parameters remain constant throughout the analysis for the bottom resonator. However, in case of top resonator, the line width is same i.e. $w=4 \mu \mathrm{m}$, but the top capacitive gap $\left(g_{2}\right)$ is varied from $2 \mu \mathrm{m}$ to $28 \mu \mathrm{m}$. The inter resonator separation (S) is varied from $2 \mu \mathrm{m}$ to $14 \mu \mathrm{m}$ in the step size of $4 \mu \mathrm{m}$.

For the fabrication of samples, we used conventional photolithography technique in the clean room environment. The samples were fabricated on a $500 \mu m$ thick high resistivity $(>5000 \mathrm{ohm}-\mathrm{cm})$ silicon substrate. Followed by photolithography, a $200 \mathrm{~nm}$ thick aluminum was deposited by using a thermal evaporator, after which a lift-off process enabled the formation of the SRR array. Optical microscope images of the fabricated samples are shown in Fig. 1(b) with the detailed geometric dimensions. The measurements were carried out using a typical $8 \mathrm{f}$ confocal terahertz time-domain spectroscopy (THz-TDS) system consisting of a photoconductive antenna transmitter and a receiver. The metallic antenna on GaAs chip is $\mathrm{dc}$ biased at $70 \mathrm{~V}$ and excited by $130 \mathrm{fs}$ optical pulses with a wavelength of $800 \mathrm{~nm}$ and a repetition rate of $80 \mathrm{MHz}$ from a Ti: sapphire oscillator laser system generating $\mathrm{THz}$ pulses with a bandwidth of $0.2-3 \mathrm{THz}$. The polarization of the incident $\mathrm{THz}$ electric field is aligned parallel to the gap bearing arms of the SRRs in order to excite the fundamental LC resonance mode (Fig. 1(b)) based 

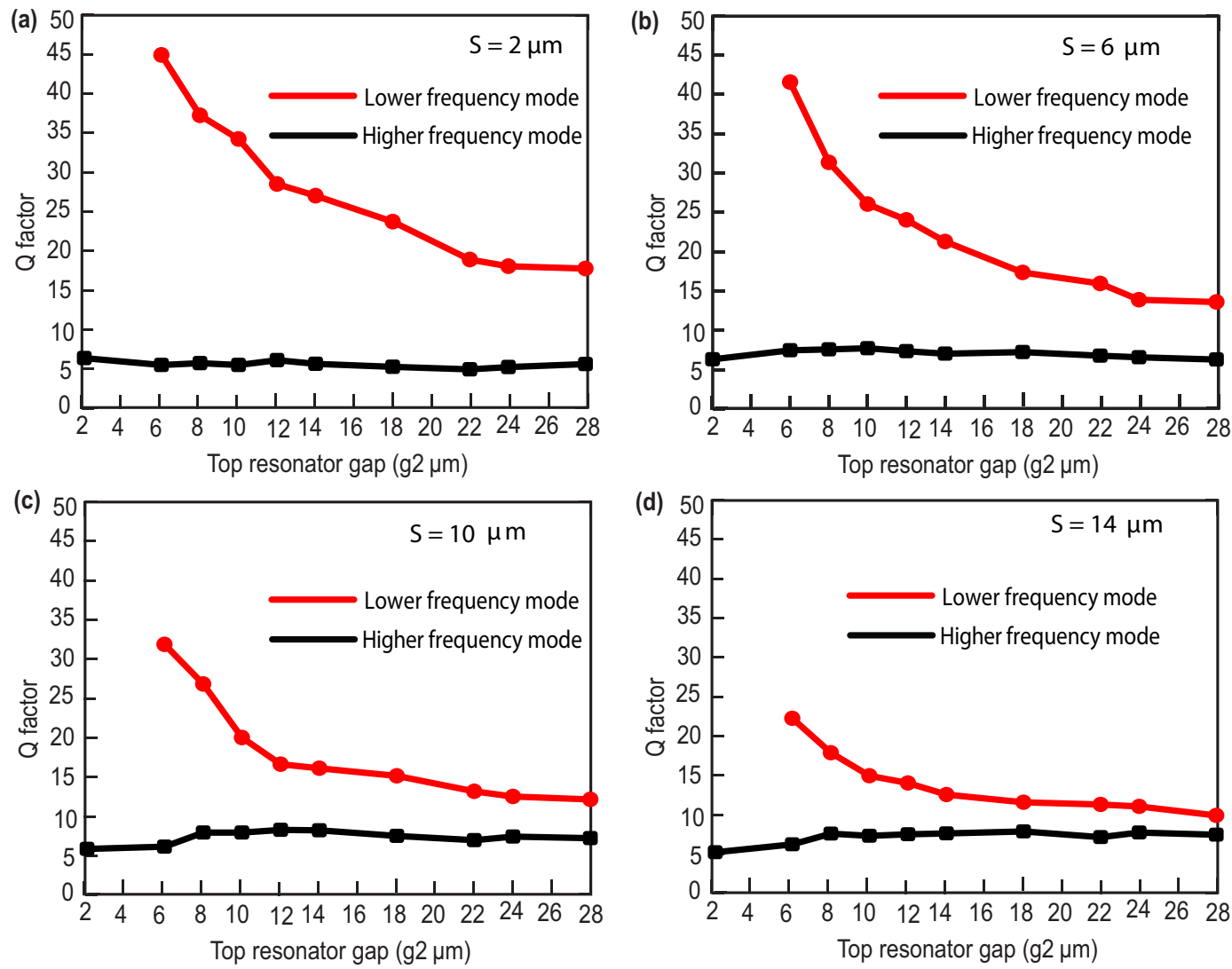

Figure 4. (a-d) Represents $\mathrm{Q}$ factor verses top resonator gap plots for $\mathrm{S}=2,6,10$ and $14 \mu \mathrm{m}$ cases respectively.

on the experiments, $\mathrm{THz}$ signal was measured in the time domain after transmitting through the metamaterial samples. It was Fourier transformed to obtain the frequency domain spectra, which is further normalized with the signal from a bare silicon substrate of the same thickness (as reference) as used in the metamaterials samples. All the measurements were done at room temperature and in a dry nitrogen atmosphere in order to mitigate the effect due to water vapor absorption. We have studied four different sets of samples corresponding to four different separations (S) between SRRs with a fixed bottom gap $\left(g_{1}\right)$, while the top resonator gap $\left(g_{2}\right)$ is changed gradually as indicated in Fig. 1(a). The separation between the resonators is symbolized by $\mathrm{S}$ in Y direction. In this study, different values of $S$ are considered to be as 2, 6, 10 and $14 \mu \mathrm{m}$. We have examined the response of $\mathrm{THz}$ transmission through the proposed design for various capacitive gaps $\left(g_{2}=2,6,10,14,18,22,26\right.$ and $\left.28 \mu \mathrm{m}\right)$ of the top resonator for four different separations ( $S$ ) both experimentally and numerically. For our numerical study, commercially available numerical software, CST Microwave Studio is employed and tetrahedral meshing is adopted for simulating the metamaterials geometry. The boundary conditions are taken as periodic in the full wave numerical simulations. We have used waveguide ports as the source and detector. The results of the $\mathrm{THz}$ transmission through the planar $\mathrm{THz}$ metamaterial systems are discussed elaborately in the next section.

\section{Discussions}

The $\mathrm{THz}$ transmission results through the fabricated metamaterial samples for four different separations (S) are shown in Fig. 2. In Fig. 2(a), the separation (S) between top and bottom SRRs is fixed at $2 \mu m$ and the top SRR gap $\left(g_{2}\right)$ is varied for $6 \mu \mathrm{m}, 26 \mu \mathrm{m}$ and $28 \mu \mathrm{m}$. In case of $g_{2}=6 \mu \mathrm{m}$, high frequency resonance mode is observed at around $0.52 \mathrm{THz}$ and low frequency mode at $0.41 \mathrm{THz}$. As the top resonator gap changes to $28 \mu \mathrm{m}$, we observed a blue shift in the higher order mode i.e. frequency shifts to $0.6 \mathrm{THz}$ while the low frequency resonance remains almost unperturbed. In case of $6 \mu \mathrm{m}$ separation, high frequency mode appears at $0.5 \mathrm{THz}$ and the low frequency mode appears around $0.44 \mathrm{THz}$. As the top gap $\left(g_{2}\right)$ increases, the high frequency mode goes through blue shifting just like the previous case. Similar trends are observed for separations $10 \mu \mathrm{m}$ and $14 \mu \mathrm{m}$. We have performed experiments to validate our results which are plotted in dashed lines in Fig. 2 along with the numerical simulations. Note that the resonance frequencies in the experiments follow simulations well however the linewidths are not in good match. The difference in the line-width of the resonance is primarily due to low spectral resolution of measurement, which is limited by the Fabry-Perot reflection pulse from the rear surface of the substrate. In order to develop a comprehensive understanding of near-field coupling in the proposed metamaterials, we simulated the coupled structures for several top resonator gap for all the four separations. The results are shown in Fig. 3 using contour plots. In Fig. 3, X axis is taken as frequency (THz), top resonator gap $\left(g_{2}\right)$ is plotted in Y axis and the color bar represents magnitude of transmission. Figure 3(a) shows the terahertz transmission for the case of separation, $\mathrm{S}=2 \mu \mathrm{m}$, whereas, Fig. 3(b-d) shows the transmission results when the separations between the coupled 
(a)

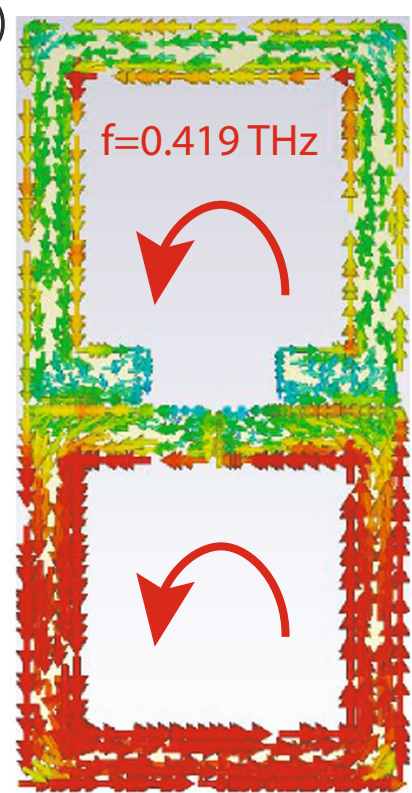

(c)

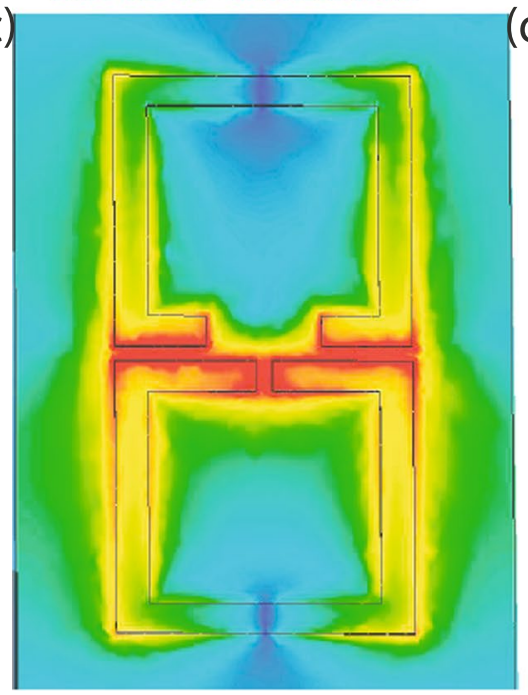

(b)

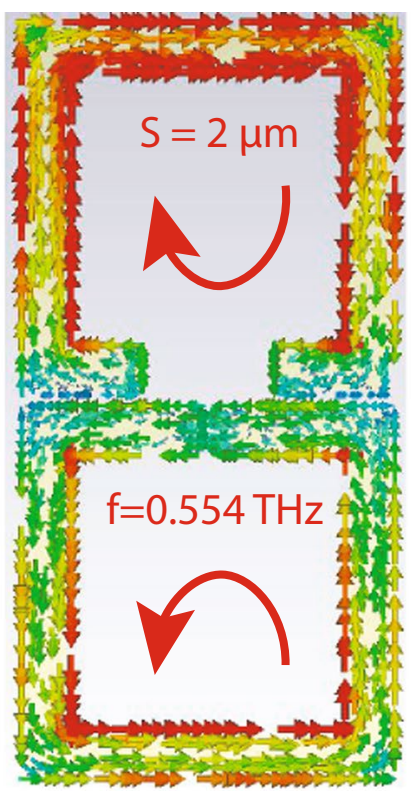

Max.

(d)

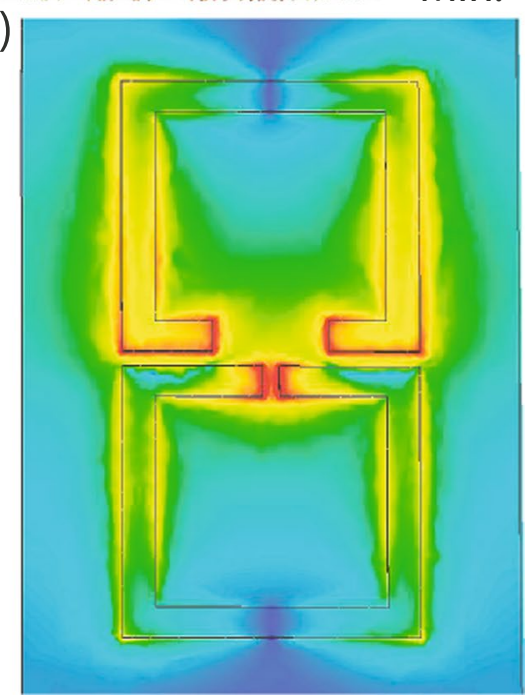

Figure 5. (a and b) Represent the surface current profile of $S=2 \mu \mathrm{m}$ case for $g_{1}=2 \mu \mathrm{m}, g_{2}=14 \mu \mathrm{m}$ at $\mathrm{f}=0.419 \mathrm{THz}$ and $\mathrm{f}=0.554 \mathrm{THz}$. (c and $\mathbf{d}$ ) Represent the electric field profiles for the same cases.

SRRs are $\mathrm{S}=6,10$ and $14 \mu \mathrm{m}$, respectively. These plots clearly indicates a resonance split when gap $g_{2}$ no longer equal to $g_{1}$. As $g_{2}$ increases, the split becomes prominent. We attribute the split in resonance to the strong coupling between the resonators primarily. The higher mode suffers clear blue shifting and this is due to the reduced capacitance of the top resonator where $g_{2}$ is increasing leading to reduction split gap capacitance of the top resonator.

In order to understand the device performance further, we have calculated the quality factor for both the resonance modes. Quality factor which is typically defined as

$$
Q=\frac{f_{r}}{\Delta f}
$$

(the ratio between the resonance frequency $f_{r}$ and the bandwidth $\Delta f$ determined at the full width at $\frac{1}{\sqrt{2}}$ maxima in case of amplitude transmission). We have calculated quality factor for all four different separations and for each separation, we have considered top resonator gap $g_{2}=2,6,8,10,12,14,18,22,24$ and $28 \mu \mathrm{m}$. To evaluate quality factor, first we have calculated the full width of each resonance mode at $\frac{1}{\sqrt{2}}$ maxima. For $\mathrm{S}=2 \mu \mathrm{m}$ case, when $g_{1}=g_{2}=2 \mu \mathrm{m}$, we have only one resonance mode at $0.5024 \mathrm{THz}$ with full width $0.08 \mathrm{THz}$ and the corresponding $\mathrm{Q}$ factor is 6.28 and for $g_{2}=6 \mu \mathrm{m}$ case, we have two resonance peaks, first resonance peak at $0.405 \mathrm{THz}$ with $\Delta f=0.009 \mathrm{THz}$ and corresponding Q factor is $\approx 45$. The second resonance peak appears at $0.524 \mathrm{THz}$ with $\Delta f=0.1 \mathrm{THz}$ and corresponding Q factor turns out to be 5.24. Similarly, we have calculated Q factor for $g_{2}=10$, 14, 18, 22 and $28 \mu \mathrm{m}$ and results are shown in Fig. 4(a). For $\mathrm{S}=6,10$ and $14 \mu \mathrm{m}$ also, the calculated Q factors are shown in Fig. 4(b-d), respectively. In all the cases, we observed lower frequency mode has higher $Q$ factor 
(a)

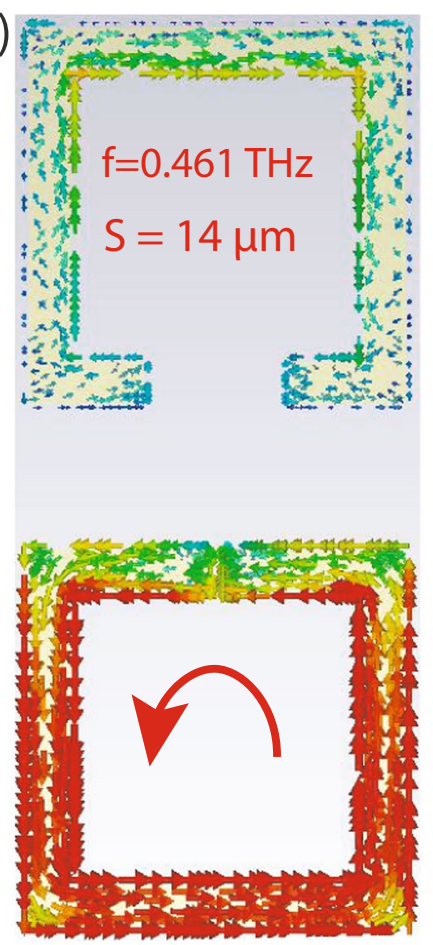

(c)

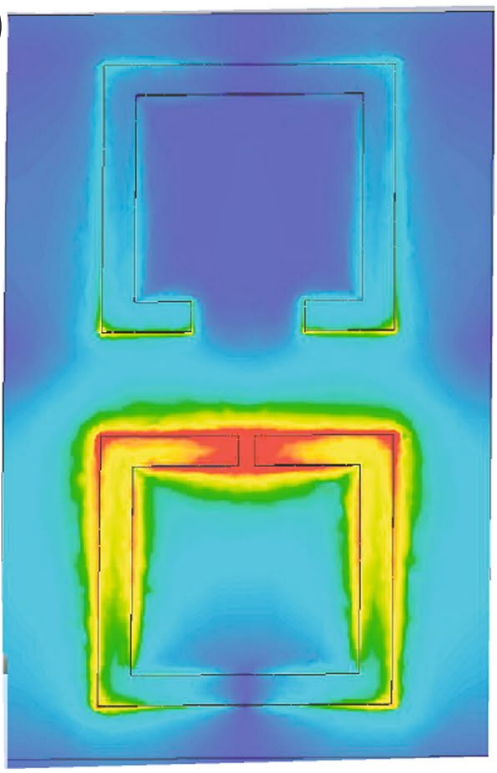

(b)

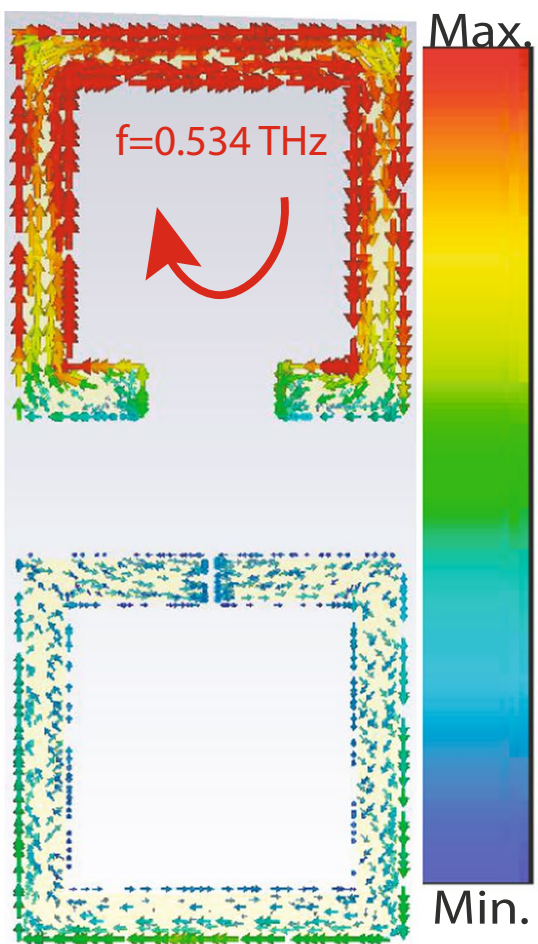

(d)

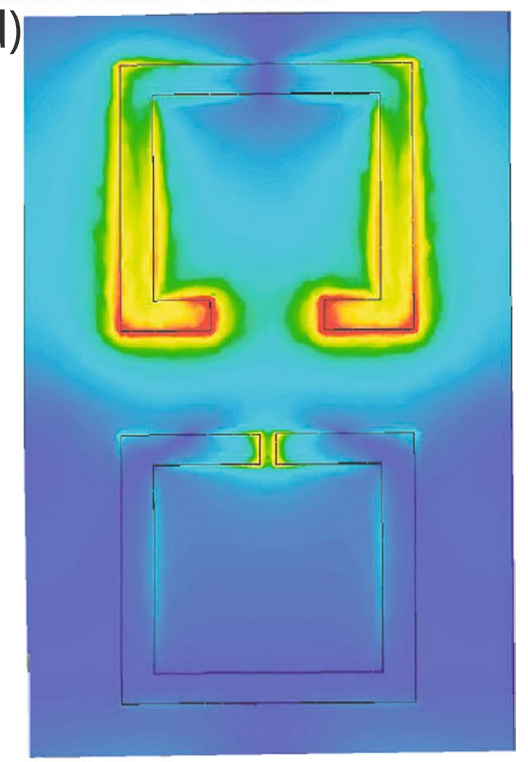

Figure 6. ( $\mathbf{a}$ and $\mathbf{b})$ Represent the surface current profile of $S=14 \mu \mathrm{m}$ case for $g_{1}=2 \mu \mathrm{m}, g_{2}=14 \mu \mathrm{m}$ at $\mathrm{f}=0.461 \mathrm{THz}$ and $\mathrm{f}=0.534 \mathrm{THz}$. (c and $\mathbf{d}$ ) Represent the electric field profiles for the same cases.

compared to the higher frequency mode. In case of minimum separation $(\mathrm{S}=2 \mu \mathrm{m})$ the $\mathrm{Q}$ factor observed is maximum. In order to validate our physical explanations, we have monitored the induced surface current profiles and electric field profiles for strongly coupled regime (Fig. 5). In case of $S=2 \mu \mathrm{m}$ separation with asymmetric split gaps in the resonators, surface currents are shown corresponding to lower and higher resonance modes, see Fig. 5(a,b). The induced surface currents in the resonators are in phase in case of lower resonance mode and $180^{\circ}$ out of phase in case of higher resonance mode. This clearly indicates that the resonators are strongly coupled through electric field lines or in other words the resonators are capacitively coupled through the split gaps. In order to validate this explanation, we have further simulated the electric field profiles at the split resonance dips as shown in Fig. 5(c,d). Electric field profiles clearly indicate that the resonators are strongly coupled at the split resonances. This also explains the trend in $\mathrm{Q}$ factor along with resonance frequency shift. $\mathrm{S}=2 \mu \mathrm{m}$ (Fig. $4 \mathrm{a}$ ) signifying strongly coupled regime, the resonators are strongest coupled therefore leading to maximum deviation in $\mathrm{Q}$ factor along with maximum shift in frequency between the resonance dips (Figs 2(a) and 3(a)). However as we move to weakly coupled regime, for example $\mathrm{S}=14 \mu \mathrm{m}$, the difference in Q factor between the modes are lesser. Because of weak capacitive interactions between the resonators the resonance dips are also not deviated from 
their intrinsic or uncoupled positions. In order to validate this point, we have further simulated the surface current distributions and electric field distributions of the resonators at weakly coupled regime $(S=14 \mu m)$ (Fig. 6). One may note that the individual resonators are strongly excited at the resonance dips signifying the coupling as weak or almost absent. At the low frequency mode, the lower resonator is excited to its fundamental mode (Fig. 6(a)). Similarly at the higher frequency mode the top resonator is excited to its fundamental mode (Fig. 6(b)). It clearly shows that the resonators are very weakly coupled and therefore resonating close to their intrinsic resonance frequencies.

\section{Conclusions}

In this work, we have examined the ability to tune resonance behavior via near field capacitive coupling in planar terahertz metamaterials. This has been achieved by manipulating the near field electric interactions via changing one resonator split gap with respect to the other resonator split gap for several inter resonator separations. Introducing asymmetry by changing the split gap in one resonator with respect to the other resonator, results in the split in the fundamental resonance mode when operated in the strong near field coupled regime. The split occurs because of the strong near field capacitive/electric interactions between the resonators. We have further calculated $\mathrm{Q}$ factor for the lower and higher resonance modes for different inter resonator separations. We observed that the lower resonance mode has significantly higher $\mathrm{Q}$ factor compared to the higher frequency resonance mode. We believe that the higher $\mathrm{Q}$ factor observed in the lower frequency resonance mode is due to the strong electric coupling between the resonators which results in larger effective inductance. In near field interaction of the resonators, we also observed a blue shift in the higher frequency split resonance. This is attributed to the enhanced split gap size leading to reduced capacitance value. The modulation of resonances in capacitive coupled planar terahertz metamaterial systems has great potential in manipulating and controlling electromagnetic waves which can ultimately result in novel applications for terahertz frequency domain such as designing sensors, antennas, modulators, switches etc.

\section{Methods}

We have fabricted MM samples using conventional photolithography technique in the clean room environment and characterized using typical $8 \mathrm{f}$ confocal terahertz time-domain spectroscopy (THz-TDS) system consisting of a photoconductive antenna transmitter and a receiver. We have used finite element frequency domain solver for the simulations in CST Microwave Studio package.

\section{References}

1. Tonouchi, M. Cutting-edge terahertz technology. Nature photonics. 1, 97 (2007).

2. Otsuji, T. et al. Graphene-based devices in terahertz science and technology. Journal of Physics D: Applied Physics 45, 303001 (2012).

3. Knap, W. et al. Nanometer size field effect transistors for terahertz detectors. Nanotechnology 24(21), 214002 (2013).

4. Docherty, C. J. \& Johnston, M. B. Terahertz properties of graphene. Journal of Infrared, Millimeter, and Terahertz Waves. 33(8), 797-815 (2012)

5. Singh, R. et al. Ultrasensitive terahertz sensing with high-Q Fano resonances in metasurfaces. Applied Physics Letters 105(17), 171101 (2014).

6. Islam, M. et al. Role of resonance modes on terahertz metamaterials based thin film sensors. Scientific reports. 7(1), 7355 (2017).

7. Jain, A., Tassin, P., Koschny, T. \& Soukoulis, C. M. Large quality factor in sheet metamaterials made from dark dielectric meta-atoms. Physical review letters 112(11), 117403 (2014).

8. Duan, G. et al. Identifying the perfect absorption of metamaterial absorbers. Physical Review B. 97(3), 035128 (2018).

9. Basharin, A A., Chuguevsky, V., Volsky, N., Kafesaki, M. \& Economou, E. N. Extremely high Q-factor metamaterials due to anapole excitation. Physical Review B. 95, 035104 (2017).

10. Manjappa, M. et al. Tailoring the slow light behavior in terahertz metasurfaces. Applied Physics Letters 106, 181101 (2015).

11. Xu, N., Singh, R. \& Zhang, W. High-Q lattice mode matched structural resonances in terahertz metasurfaces. Applied Physics Letters 109, 021108 (2016).

12. Shelby, R. A., Smith, D. R. \& Schultz, S. Experimental verification of a negative index of refraction. Science 292(5514), 77-79 (2001).

13. Fang, N., Lee, H., Sun, C. \& Zhang, X. Sub-diffraction-limited optical imaging with a silver superlens. Science 308, 534-537 (2005).

14. Pendry, J. B., Schurig, D. \& Smith, D. R. Controlling electromagnetic fields. science 312, 1780-1782 (2006).

15. Orazbayev, B., Estakhri, N. M., Beruete, M. \& Alù, A. Terahertz carpet cloak based on a ring resonator metasurface. Physical Review B 91, 195444 (2015)

16. Liu, H. et al. Magnetic plasmon hybridization and optical activity at optical frequencies in metallic nanostructures. Physical Review $B$ 76, 073101 (2007).

17. Liu, N., Liu, H., Zhu, S. \& Giessen, H. Stereometamaterials. Nature Photonics 3, 157 (2009).

18. Singh, R., Rockstuhl, C., Lederer, F. \& Zhang, W. Coupling between a dark and a bright eigenmode in a terahertz metamaterial. Phys. Rev. B 79, 085111 (2009).

19. Reiten, M. T. et al. Resonance tuning behavior in closely spaced inhomogeneous bilayer metamaterials. Applied Physics Letters $\mathbf{9 8 ,}$ 131105 (2011).

20. Chowdhury, D. R., O’Hara, J. F., Taylor, A. J. \& Azad, A. K. Orthogonally twisted planar concentric split ring resonators towards strong near field coupled terahertz metamaterials. Appl. Phys. Lett. 104, 101105 (2014).

21. Li, J. et al. Mechanically tunable terahertz metamaterials. Applied Physics Letters 102, 121101 (2013).

22. Chen, H.-T. et al. Active terahertz metamaterial devices. Nature 444, 597-600 (2006).

23. Ekmekci, E. et al. Frequency tunable terahertz metamaterials using broadside coupled split-ring resonators. Physical Review B 83, 193103 (2011).

24. Dietze, D., Unterrainer, K. \& Darmo, J. Role of geometry for strong coupling in active terahertz metamaterials. Physical Review B 87, 075324 (2013).

25. Chowdhury, D. R. et al. A broadband planar terahertz metamaterial with nested structure. Optics Express 19, 15817-15823 (2011).

26. Chowdhury, D. R. et al. Dynamically reconfigurable terahertz metamaterial through photo-doped semiconductor. Appl. Phys. Lett. 99, 231101 (2011)

27. Yang, Y. et al. Hyperbolic spoof plasmonic metasurfaces. NPG Asia Materials 9, e428 (2017).

28. Jing, L. et al. Chiral metamirrors for broadband spin-selective absorption. Applied Physics Letters 110, 231103 (2017).

29. Wang, Z. et al. Manipulating Smith-Purcell Emission with Babinet Metasurfaces. Physical review letters 117, 157401 (2016).

30. Wang, Z. et al. Origami-Based Reconfigurable Metamaterials for Tunable Chirality. Advanced Materials 29, 1700412 (2017). 
31. Jing, L. et al. Gradient Chiral Metamirrors for Spin-Selective Anomalous Reflection. Laser \& Photonics Reviews 11, 1700115 (2017).

32. Chen, H., Wu, B.-I., Zhang, B. \& Kong, J. A. Electromagnetic wave interactions with a metamaterial cloak. Physical Review Letters 99, 063903 (2007).

33. Peng, L. et al. Experimental observation of left-handed behavior in an array of standard dielectric resonators. Physical review letters 98, 157403 (2007).

34. Pendry, J. B., Holden, A. J., Robbins, D. J. \& Stewart, W. J. Magnetism from conductors and enhanced nonlinear phenomena. IEEE transactions on microwave theory and techniques 47, 2075-2084 (1999).

35. Cai, W. \& Shalaev, V. M. Optical metamaterials. Springer 10, 6011 (2010).

36. Zhao, J. et al. A tunable metamaterial absorber using varactor diodes. New Journal of Physics 15, 043049 (2013).

37. Engheta, N. \& Ziolkowski, R. W. Metamaterials: physics and engineering explorations. John Wiley \& Sons (2006).

38. Chowdhury, D. R., Xu, N., Zhang, W. \& Singh, R. Resonance tuning due to Coulomb interaction in strong near field coupled metamaterials. Journal of Applied Physics 118, 023104 (2015).

39. Rao, S. J. M., Kumar, D., Kumar, G. \& Chowdhury, D. R. Probing the near field Inductive Coupling in Broadside Coupled Terahertz Metamaterials. IEEE Journal of Selected Topics in Quantum Electronics 23, 1-7 (2017).

40. Schurig, D., Mock, J. J. \& Smith, D. R. Electric-field-coupled resonators for negative permittivity metamaterials. Applied physics letters 88, 041109 (2006).

41. Sheng, Z. \& Varadan, V. V. Tuning the effective properties of metamaterials by changing the substrate properties. Journal of applied physics 101, 014909 (2007).

42. Zhao, Q. et al. Electrically tunable negative permeability metamaterials based on nematic liquid crystals. Applied physics letters $\mathbf{9 0 ,}$ 011112 (2007).

43. Kowerdziej, R., Jaroszewicz, L., Olifierczuk, M. \& Parka, J. Experimental study on terahertz metamaterial embedded in nematic liquid crystal. Applied physics letters 106, 092905 (2015).

44. Han, J., Lakhtakia, A. \& Qiu, C.-W. Terahertz metamaterials with semiconductor split-ring resonators for magnetostatic tunabilityl. Optics Express 16, 14390-14396 (2008).

45. Harris, S. E., Field, J. E. \& Imamoğlu, A. Nonlinear optical processes using electromagnetically induced transparency. Physical Review Letters 64, 1107 (1990).

46. Ekmekci, E., Topalli, K., Akin, T. \& Turhan-Sayan, G. A tunable multi-band metamaterial design using micro-split SRR structures. Optics express 17, 16046-16058 (2009).

47. Padilla, W. J. et al. Electrically resonant terahertz metamaterials: Theoretical and experimental investigations. Physical Review B 75, 041102 (2007).

48. Tao, H. et al. Reconfigurable terahertz metamaterials. Physical review letters 103, 147401 (2009).

49. Li, C., Chang, C.-C., Zhou, Q., Zhang, C. \& Chen, H.-T. Resonance coupling and polarization conversion in terahertz metasurfaces with twisted split-ring resonator pairs. Optics Express 25, 25842-25852 (2017).

50. Al-Naib, I. et al. Conductive coupling of split ring resonators: a path to THz metamaterials with ultrasharp resonances. Physical review letters 112, 183903 (2014).

51. Ma, S., Xiao, S. \& Zhou, L. Resonant modes in metal/insulator/metal metamaterials: An analytical study on near field couplings. Physical Review B 93, 045305 (2016).

52. Bai, Z. \& Huang, G. Plasmonic solitons and dromions via plasmon-induced transparency. Journal of Physics: Conference Series 752 , $012005(2016)$

53. Azad, A. K., Taylor, A. J., Smirnova, E. \& O'Hara, J. F. Characterization and analysis of terahertz metamaterials based on rectangular split-ring resonators. Applied Physics Letters 92, 01111 (2008).

54. Srivastava, Y. K. et al. Ultrahigh-Q Fano Resonances in Terahertz Metasurfaces: Strong Influence of Metallic Conductivity at Extremely Low Asymmetry. Advanced Optical Materials 4, 457-463 (2016).

55. Rao, S. J. M., Kumar, D., Kumar, G. \& Chowdhury, D. R. Modulating the Near Field Coupling through Resonator Displacement in Planar Terahertz Metamaterials. Journal of Infrared, Millimeter, and Terahertz Waves 38, 124-134 (2017).

56. Chowdhury, D. R. et al. Tailored resonator coupling for modifying the terahertz metamaterial response. Tailored resonator coupling for modifying the terahertz metamaterial response 19, 10679-10685 (2011).

\section{Acknowledgements}

G.K. gratefully acknowledge the financial support from the SERB, Department of Science and Technology, India (SB/FTP/PS-051/2014). DRC gratefully acknowledge the financial support from the SERB, Department of Science and Technology, India (EMR/2015/001339). SJM Rao would like to acknowledge the financial support from the Ministry of Human Resource Development, Government of India for a research fellowship.

\section{Author Contributions}

Author D.R.C. proposed the idea. Authors D.R.C. and G.K. supervised the work. Authors S.J.M. Rao and Yogesh Kumar Srivastava performed the simulations and expermental work, respectively. All the authors contributed in analysing the data and writing the manuscript.

Additional Information

Competing Interests: The authors declare no competing interests.

Publisher's note: Springer Nature remains neutral with regard to jurisdictional claims in published maps and institutional affiliations.

Open Access This article is licensed under a Creative Commons Attribution 4.0 International License, which permits use, sharing, adaptation, distribution and reproduction in any medium or format, as long as you give appropriate credit to the original author(s) and the source, provide a link to the Creative Commons license, and indicate if changes were made. The images or other third party material in this article are included in the article's Creative Commons license, unless indicated otherwise in a credit line to the material. If material is not included in the article's Creative Commons license and your intended use is not permitted by statutory regulation or exceeds the permitted use, you will need to obtain permission directly from the copyright holder. To view a copy of this license, visit http://creativecommons.org/licenses/by/4.0/.

(C) The Author(s) 2018 